\title{
Atividade de microrganismos solubilizadores de fosfatos na presença de nitrogênio, ferro, cálcio e potássio(1)
}

\author{
Germano Nunes Silva Filho(2) e Caio Vidor ${ }^{(3)}$
}

Resumo - A capacidade e o potencial de solubilização de 21 isolados de microrganismos solubilizadores de fosfatos (Bacillus, Pseudomonas, Enterobacteriaceae, Penicillium, Aspergillus e Paecilomyces) foram avaliados em cultivos em meio de cultura Glicose-Extrato de Levedura contendo diferentes fosfatos ( $\mathrm{Ca}, \mathrm{Al}$ ou $\mathrm{Fe}$ ), na presença de fontes de $\mathrm{N}$ (peptona, amônio e nitrato) e teores de $\mathrm{Fe}, \mathrm{Ca}$ e $\mathrm{K}$. $\mathrm{O}$ crescimento e a atividade solubilizadora variaram em função do tipo de microrganismo e dos fatores nutricionais. Em relação às fontes de N, a presença de amônio favoreceu a solubilização em seis isolados; destes, três solubilizaram somente nesta fonte. O nitrato diminuiu a atividade solubilizadora, reduzindo ou inibindo a solubilização. Para a maioria dos microrganismos, a atividade solubilizadora não foi afetada pelas variações nos teores de ferro. Baixos teores de Ca e $\mathrm{K}$ limitaram o crescimento de cinco isolados que apresentam características de amplo crescimento (Aspergillus). Em dois desses isolados, a solubilização de fosfato de Ca foi favorecida. Variações na capacidade e no potencial de solubilização dos microrganismos, em resposta às condições do meio de cultura, indicam que o processo ocorre com eficiência variável ou sugerem a presença de diferentes mecanismos de solubilização.

Termos para indexação: fósforo, microrganismos do solo, solubilização, nitrato, amônio.

Phosphate solubilizing activity of microorganisms in the presence of nitrogen, iron, calcium and potassium

\begin{abstract}
Twenty-one isolates of phosphate solubilizing microorganisms (Bacillus, Pseudomonas, Enterobacteriaceae, Penicillium, Aspergillus and Paecilomyces) were evaluated for their solubilizing capacity and potential in broth media (Glucose-Yeast Extract) amended with different types of phosphates $(\mathrm{Ca}, \mathrm{Al}$ or $\mathrm{Fe})$, in the presence of $\mathrm{N}$ sources (peptone, ammonium and nitrate) as well as concentrations of $\mathrm{Fe}, \mathrm{Ca}$ and potassium. Microbial growth and phosphate solubilizing activity varied according to the microorganism and the nutrient supplied. Ammonium increased solubilization activity of six isolates and three of these solubilized only in this source. Nitrate decreased solubilizing activity and in some instances, completely inhibited solubilization. In general, the solubilizing activity of microbial isolates was not affected by different concentrations of iron. Low concentrations of Ca and $\mathrm{K}$ limited the growth of five isolates that characteristically form large colonies (Aspergillus). Solubilization of calcium phosphate was increased by two of these isolates. Variations in the phosphate solubilizing capacity and potential of microorganisms as a response to the conditions in the growth medium indicate that the process occurs with variable efficiency or suggest the presence of different mechanisms of solubilization.
\end{abstract}

Index terms: phosphorus, soil, microorganisms, solubilization, nitrate, ammonium.

(1) Aceito para publicação em 30 de maio de 2001.

Extraído da Tese de Doutorado apresentada pelo primeiro autor à Universidade Federal do Rio Grande do Sul (UFRGS), Porto Alegre, RS.

(2) Universidade Federal de Santa Catarina, Centro de Ciências Biológicas, Dep. de Microbiologia e Parasitologia, Caixa Postal 476, CEP 88010-970 Florianópolis, SC. Bolsista da Capes/PICD. E-mail: germano@ccb.ufsc.br

(3)UFRGS, Fac. de Agronomia, Dep. de Solos, Caixa Postal 776 , CEP 90001-970 Porto Alegre, RS. E-mail: vidor@uol.com.br

\section{Introdução}

Os microrganismos solubilizadores de fosfatos inorgânicos desempenham importante papel no suprimento de P para as plantas. Este fato tem despertado a atenção para a utilização desses microrganismos como inoculante comercial ou no manejo de suas populações como forma de promover uma melhor utilização do P existente no solo ou do adicionado como fertilizante. 
O uso desses microrganismos depende do conhecimento de suas características, entre as quais, a capacidade de solubilização é uma das mais importantes no processo de seleção. Ela varia com o microrganismo e as condições do ambiente. Entre os fatores ambientais, o tipo de fosfato e a fonte de C estão entre os mais estudados (Silva Filho \& Vidor, 2000). No entanto, a fonte de $\mathrm{N}$ e teores de nutrientes inorgânicos podem ter participação decisiva no processo. $\mathrm{O}$ efeito da fonte de $\mathrm{N}$ tem sido relacionado ao balanço de íons absorvidos (Fernandes \& Souza, 1990; Darrah, 1993). De modo geral, a solubilização aumenta com a absorção de fontes amoniacais e diminui com as nítricas. No entanto, existem exceções, como a constatada por Lapeyrie et al. (1991) com um fungo ectomicorrízico da espécie Paxillus involutus, cuja solubilização foi estimulada pela presença de nitrato.

$\mathrm{O} \mathrm{Fe}$, o $\mathrm{Ca}$ e o $\mathrm{K}$ apresentam funções que sugerem as suas participações no processo de solubilização de fosfatos. A participação do Fe na constituição de várias enzimas faz com que sua disponibilidade esteja relacionada ao controle de vias metabólicas (Bagg \& Neilands, 1987; Marschner, 1995). A enzima aconitase é responsável pela isomerização do citrato, reação necessária à continuidade do ciclo dos ácidos tricarboxílicos. Diminuição na atividade desta enzima provoca acúmulo de ácidos orgânicos (Marschner, 1995). Esses ácidos, ao serem liberados no meio, favorecem a solubilização de fosfatos.

$\mathrm{O}$ Ca como nutriente é necessário à atividade enzimática e à estrutura da membrana (Griffin, 1994), e sua deficiência favorece a solubilização de fosfatos deste elemento (MacKay et al., 1986; Robinson \& Syers, 1990). O K participa da atividade enzimática, do metabolismo de carboidratos e do balanço iônico (Griffin, 1994) e sua deficiência tem provocado maior liberação de ácidos orgânicos (Paris et al., 1996).

A análise do comportamento dos isolados nas diferentes condições nutricionais poderá fornecer informações para a maximização do processo. Isto poderá ser utilizado para melhorar o rendimento nas condições de meio de cultura ou para a máxima eficiência no campo. Além disso, fornecerão dados que poderão contribuir para o entendimento dos mecanismos utilizados na solubilização. A solubilização é freqüentemente associada à produção de ácidos (Cunningham \& Kuiack, 1992; Kim et al., 1997), no entanto, outros mecanismos podem ocorrer (Illmer \& Schinner, 1992).

O objetivo deste trabalho foi avaliar o comportamento de microrganismos solubilizadores de fosfatos em resposta a fontes de $\mathrm{N}$ e teores de $\mathrm{Fe}, \mathrm{Ca}$ e $\mathrm{K}$ no meio de cultura.

\section{Material e Métodos}

Foram utilizados 21 isolados (Tabela 1) pertencentes à coleção de microrganismos solubilizadores de fosfatos do Laboratório de Microbiologia do Solo do Departamento de Microbiologia e Parasitologia do Centro de Ciências Biológicas da Universidade Federal de Santa Catarina, classificados em gêneros por Silva Filho (1998) e selecionados por suas características morfofisiológicas e por sua capacidade de solubilizar fosfatos (Silva Filho \& Vidor, 2000).

Os isolados foram avaliados em meio de cultura GEL (Sylvester-Bradley et al., 1982) com 0,3\% de extrato de levedura e suplementado com uma das três fontes de $\mathrm{P}$ $\left(\mathrm{CaHPO}_{4}, \mathrm{AlPO}_{4}\right.$ ou $\left.\mathrm{FePO}_{4}\right)$, na proporção de $0,89 \mathrm{~g} \mathrm{~L}^{-1}$ de P. O fosfato de Ca foi obtido pela adição de $1 \mathrm{~mL}$ de uma solução de $\mathrm{K}_{2} \mathrm{HPO}_{4}$ a $5 \%$ e $1 \mathrm{~mL}$ de uma solução de $\mathrm{CaCl}_{2}$ a $10 \%$ por $10 \mathrm{~mL}$ de meio. $\mathrm{O}$ fosfato de $\mathrm{Al}$ foi adicionado na forma de suspensão na proporção de $3,5 \mathrm{~g} \mathrm{~L}^{-1}$ de meio. O fosfato de $\mathrm{Fe}$, por se encontrar na forma de grânulos, foi moído e passado em peneira de $0,053 \mathrm{~mm}$ de

Tabela 1. Relação dos isolados de microrganismos solubilizadores de fosfatos.

\begin{tabular}{cl}
\hline $\mathrm{N}^{\circ}$ do isolado & Microrganismo \\
\hline 04 & Penicillium \\
05 & Penicillium \\
85 & Pseudomonas \\
86 & Aspergillus \\
89 & Penicillium \\
98 & Penicillium \\
119 & Aspergillus \\
142 & Aspergillus \\
148 & Pseudomonas \\
154 & Enterobacteriaceae (Enterobacter ou Erwinia) \\
205 & Bastonete Gram + sem esporos (não identificado) \\
238 & Penicillium \\
239 & Bacillus \\
250 & Aspergillus \\
263 & Penicillium \\
264 & Penicillium \\
293 & Paecilomyces \\
297 & Enterobacteriaceae (Enterobacter ou Erwinia) \\
303 & Penicillium \\
$310 \mathrm{~A}$ & Aspergillus \\
$310 \mathrm{~B}$ & Aspergillus \\
\hline
\end{tabular}


abertura de malha. O material peneirado foi utilizado na forma de suspensão, na proporção de 4,33 $\mathrm{g} \mathrm{L}^{-1}$. Em cada fosfato foram avaliados os efeitos de fontes de $\mathrm{N}$ e teores de $\mathrm{Fe}, \mathrm{Ca}$ e potássio.

As fontes de $\mathrm{N}$ foram avaliadas pela adição de $\mathrm{NH}_{4}{ }^{+}$, $\mathrm{NO}_{3}^{-}, \mathrm{NH}_{4}{ }^{+}+\mathrm{NO}_{3}^{-}(1: 1)$ e peptona, na proporção de 69,2 mg de $\mathrm{N} \mathrm{L}^{-1}$ de meio GEL, utilizando-se como fontes inorgânicas de $\mathrm{N}$ o cloreto de amônio e o nitrato de $\mathrm{K}$. Como testemunha, usou-se o meio GEL.

Os teores de $\mathrm{Fe}$, Ca e $\mathrm{K}$ foram testados em sete níveis, adotando-se como valor intermediário o do meio GEL. $\mathrm{O} F$ e foi utilizado na forma de cloreto férrico em escala exponencial com intervalo de magnitude de $0,5(0,45,1$, $2,23,4,96,11,04,24,6$ e $54,8 \mathrm{mg} \mathrm{L}^{-1}$ de meio).

Foram testados teores de $\mathrm{Ca}$ de 230, 900, 1.400, 2.800 , $5.000,6.900$ e $8.800 \mathrm{mg} \mathrm{L}^{-1}$ no fosfato de $\mathrm{Ca}$ e 60, 900, $1.800,2.800,3.700,5.000$ e $6.400 \mathrm{mg} \mathrm{L}^{-1}$ no fosfato de $\mathrm{Al}$ e no de $\mathrm{Fe}$. Esses valores foram obtidos após a adoção dos seguintes procedimentos: o teor de $230 \mathrm{mg} \mathrm{L}^{-1}$ foi obtido pela lavagem do precipitado por duas vezes antes de ser adicionado ao meio. Após a mistura das soluções de $\mathrm{K}_{2} \mathrm{HPO}_{4}$ e $\mathrm{CaCl}_{2}$, a suspensão foi centrifugada a $3.000 \mathrm{rpm}$ por 5 minutos. Em seguida, o sobrenadante foi desprezado, e adicionou-se água destilada para reconstituir o volume inicial, procedendo-se então à ressuspensão e nova centrifugação. Este procedimento foi repetido e finalmente uma nova ressuspensão foi produzida No segundo tratamento $\left(900 \mathrm{mg} \mathrm{L}^{-1}\right)$, foi removido $75 \%$ do sobrenadante da primeira centrifugação e adição de igual volume de água destilada. No terceiro, a remoção foi de $50 \%$. No quarto tratamento, o precipitado foi formado no meio de cultura, mediante a adição de $1 \mathrm{~mL}$ de $\mathrm{K}_{2} \mathrm{HPO}_{4}$ a $5 \%$ e $1 \mathrm{~mL}$ de $\mathrm{CaCl}_{2}$ a $10 \%$ por $10 \mathrm{~mL}$ de meio O quinto, o sexto e o sétimo tratamento foram obtidos adicionando-se, respectivamente, ao procedimento adotado no quarto tratamento, $2.000,4.000$ e $6.000 \mathrm{mg}$ de $\mathrm{Ca}$ na forma de $\mathrm{CaCl}_{2}$ por litro de meio. Quando as fontes de $\mathrm{P}$ foram os fosfatos de $\mathrm{Al}, \mathrm{Fe}$ ou a testemunha sem adição de fosfato inorgânico, os tratamentos foram preparados pela adição de $0,1.000,2.000,3.000,4.000,5.000 \mathrm{e}$ $6.000 \mathrm{mg}$ de $\mathrm{Ca}$ na forma de $\mathrm{CaCl}_{2}$ ao meio GEL

Os teores de $\mathrm{K}$ avaliados foram de 130, 700, 1.000 , $2.000,2.600,3.100$ e $3.700 \mathrm{mg} \mathrm{L}^{-1}$ no fosfato de Ca e de $30,500,1.100,1.700,2.100,2.500$ e $3.500 \mathrm{mg} \mathrm{L}^{-1}$ no fosfato de $\mathrm{Al}$ e no de $\mathrm{Fe}$. Os quatro primeiros tratamentos em cada fosfato foram obtidos de maneira semelhante à preparação dos níveis de $\mathrm{Ca}$. O quinto, o sexto e o sétimo tratamento foram obtidos adicionando-se respectivamente ao quarto tratamento $600,1.200$ e $1.800 \mathrm{mg}$ de $\mathrm{K}$ na forma de $\mathrm{KCl}$ por litro de meio. Nas demais fontes de fosfato, os diferentes níveis de $\mathrm{K}$ foram obtidos pela adição de $0,600,1.200,1.800,2.400,3.000$ e $3.600 \mathrm{mg}$ de potássio. Determinações dos teores de K e Ca solúveis no meio foram realizadas após a aplicação dos tratamentos e centrifugação (Tedesco et al., 1995)

O inóculo foi preparado adicionando-se $3 \mathrm{~mL}$ de água destilada esterilizada a culturas incubadas em meio GEL inclinado em tubos de ensaio $(120 \times 80 \mathrm{~mm})$, por 72 horas a $30^{\circ} \mathrm{C}$, seguida de agitação manual. A inoculação foi realizada com ponteira de pipeta de diâmetro aproximado de $1 \mathrm{~mm}$. Em cada placa, foram inoculados aleatoriamente sete isolados em pontos eqüidistantes. Após 72 horas de incubação a $30^{\circ} \mathrm{C}$, foi verificada a presença de área solubilizada (capacidade) e realizada a medição do diâmetro dessa área e da colônia. A partir destes dados, foi obtida a relação entre o diâmetro do halo e da colônia. O diâmetro da área solubilizada e a relação foram utilizados na avaliação do potencial de solubilização.

Cada isolado foi avaliado individualmente, em experimento conduzido em delineamento de blocos casualizados, com cinco repetições. Os dados obtidos foram submetidos à análise de variância e as médias comparadas pelo teste de Tukey a $5 \%$ de probabilidade.

\section{Resultados e Discussão}

Independentemente da fonte de $\mathrm{N}$, nenhum isolado solubilizou fosfato de $\mathrm{Fe}$, enquanto dois solubilizaram fosfato de $\mathrm{Al}$ e 17 solubilizaram fosfato de $\mathrm{Ca}$ em pelo menos uma das fontes nitrogenadas. Quatro isolados (4, 89, 119 e 148), conforme já havia sido observado por Silva Filho \& Vidor (2000), não solubilizaram nenhum tipo de fosfato.

Entre os isolados que solubilizaram fosfato de $\mathrm{Ca}$, seis apresentaram efeitos positivos do $\mathrm{N}$ amoniacal (Figura 1). Os isolados 98, 205 e 239 só solubilizaram na presença desta fonte. Com a absorção de amônio, a célula tem que promover a extrusão de prótons $\left(\mathrm{H}^{+}\right)$ para manter a sua eletroneutralidade e o transporte ativo secundário em funcionamento (Fernandes \& Souza, 1990; Haynes, 1990; Darrah, 1993), acidificando o meio e conseqüentemente solubilizando o fosfato. Além de ser de baixa eficiência (relação halo: colônia $=1$ ), este parece ser o único mecanismo que estes microrganismos apresentam. Esta idéia é reforçada pelo fato de estes três isolados não terem solubilizado fosfato em experimentos anteriores (Silva Filho \& Vidor, 2000).

Os isolados 154, 263 e 297 apresentam efeito positivo da nutrição amoniacal e negativo da nítrica (Figura 1). Quando a fonte foi exclusivamente nítrica, 
não houve solubilização. A absorção do nitrato leva ao consumo de prótons por parte da célula (Fernandes \& Souza, 1990; Neidhardt et al., 1990), ocasionando a elevação do $\mathrm{pH}$ no meio. Quando se utilizou $\mathrm{NH}_{4}^{+}+\mathrm{NO}_{3}^{-}$, houve solubilização, mas os valores do diâmetro do halo e da relação (halo:colônia) foram inferiores aos da nutrição amoniacal. Na presença das duas formas, a célula pode absorver quantidades balanceadas, de forma a não alterar o pH (Fernandes \& Souza, 1990; Darrah, 1993).

Um isolado (264) só solubilizou fosfato de Ca na presença de peptona ou quando o meio (GEL) não foi suplementado com N (Figura 1). Deficiências nutricionais podem favorecer a produção e ou a liberação de ácidos. A limitação do crescimento no meio não-suplementado pode ser uma das justificativas para a solubilização (Cunningham \& Kuiack, 1992; Illmer et al., 1995).

Dez isolados $(05,85,86,142,238,250,293,303$, 310 A e 310 B) solubilizaram fosfato de $\mathrm{Ca}$ independentemente da fonte de $\mathrm{N}$ (Figuras 1 e 2). O crescimento dos isolados 05, 85, 238, 293 e 303 não foi afetado. Apenas o isolado 303 apresentou maior relação halo:colônia na presença de $\mathrm{NO}_{3}{ }^{-}$quan-

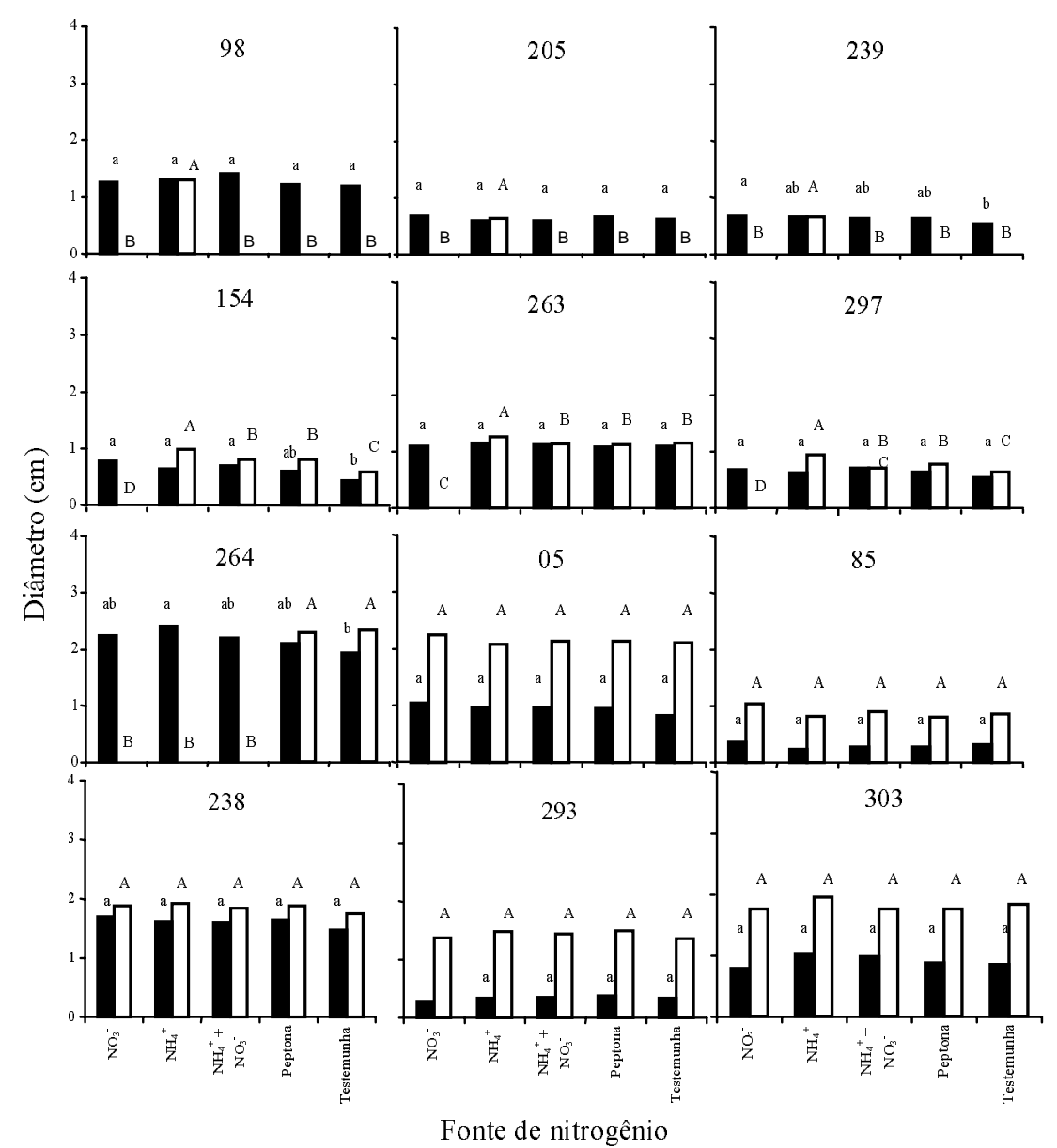

Figura 1. Efeito de fontes de nitrogênio no crescimento e na capacidade de solubilizar fosfato de cálcio dos isolados 98, 205, 239, 154, 263, 297, 264, 05, 85, 238, 293 e 303. Letras minúsculas comparam o diâmetro de colônia $(\square)$ e maiúsculas o do halo ( $\square$ ). 


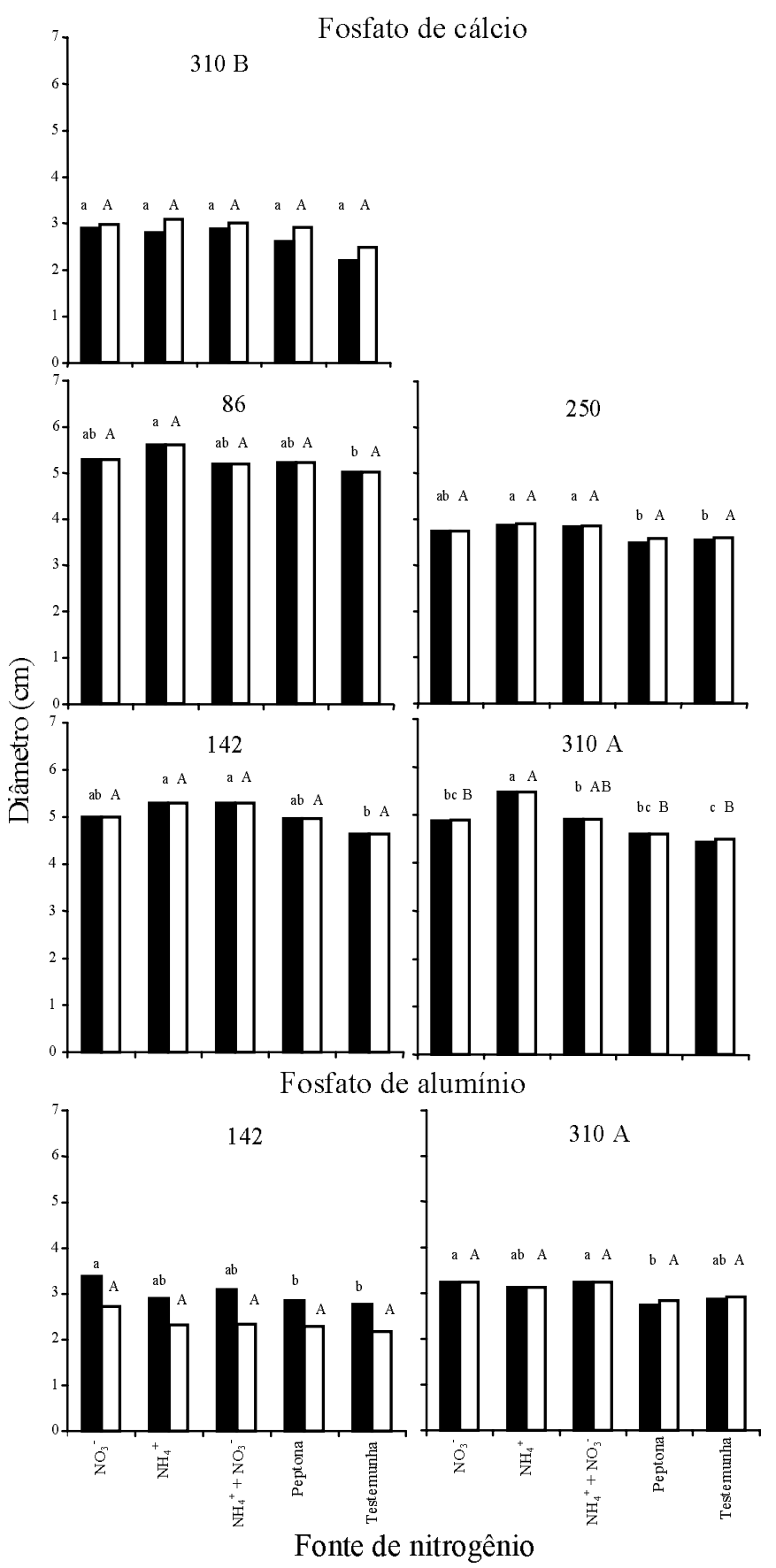

Figura 2. Efeito de fontes de $\mathrm{N}$ no crescimento e na capacidade de solubilizar fosfato de cálcio dos isolados $310 \mathrm{~B}, 86,250,142$ e $310 \mathrm{~A}$ e de alumínio dos isolados 142 e 310 A. Letras minúsculas comparam o diâmetro de colônia $(\boldsymbol{\square})$ e maiúsculas o do halo $(\square)$. 
do comparada às obtidas com $\mathrm{NH}_{4}{ }^{+} \mathrm{e} \mathrm{NH}_{4}{ }^{+}+\mathrm{NO}_{3}{ }^{-}$. Este microrganismo pode ter um metabolismo semelhante ao Paxillus involutus, onde a presença de nitrato estimula a produção de ácidos orgânicos (Lapeyrie et al., 1991; Paris et al., 1996) como uma forma de compensar a elevação do $\mathrm{pH}$ da célula devido à absorção de nitrato (Lapeyrie, 1988).

Os isolados que apresentaram colônias grandes $(86,142,250,310$ A e 310 B) tiveram o crescimento estimulado pelas fontes nitrogenadas inorgânicas (Figura 2). Apesar de o isolado $310 \mathrm{~B}$ não apresentar diferenças de crescimento entre as fontes inorgânicas, a relação halo:colônia na presença de nitrato $\left(\mathrm{NO}_{3}^{-}\right.$ou $\left.\mathrm{NH}_{4}^{+}+\mathrm{NO}_{3}^{-}\right)$foi inferior aos demais tratamentos, sugerindo o efeito de inibição pelo $\mathrm{NO}_{3}{ }^{-}$. Os resultados obtidos para o fosfato de $\mathrm{Al}$ pelos isolados 142 e $310 \mathrm{~A}$ foram semelhantes aos obtidos com o fosfato de $\mathrm{Ca}$, sugerindo que o mecanismo é provavelmente o mesmo.

Nos níveis de $\mathrm{Fe}, \mathrm{Ca}$ ou $\mathrm{K}$, sete isolados $(04,89$, $98,119,148,205$ e 239) não solubilizaram fosfatos, repetindo outros resultados obtidos por Silva Filho \& Vidor (2000). Dos 14 restantes, nenhum isolado demonstrou capacidade de solubilizar fosfato de $\mathrm{Fe}$, dois solubilizaram fosfato de $\mathrm{Al}$ e todos solubilizaram o fosfato de Ca em pelo menos um nível de $\mathrm{Fe}$, Ca ou potássio. Observou-se a formação de precipitado de fosfato de $\mathrm{Ca}$ nos experimentos com Ca e K, principalmente nos tratamentos 2 e 3 , em que o meio apresentou áreas concentradas com grumos.

A atividade solubilizadora da maioria dos microrganismos não foi afetada pelos teores de ferro. Isto pode significar que os valores utilizados não foram limitantes ou que estes não apresentam mecanismos eficientes para a solubilização deste fosfato. Dos 14 isolados que solubilizaram, dez o fizeram em todos os níveis. Destes, oito só solubilizaram fosfato de $\mathrm{Ca}$ (Figura 3). Além do fosfato de $\mathrm{Ca}$, os isolados $142 \mathrm{e}$ 310 A solubilizaram o fosfato de Al (Figura 4). Estes mesmos isolados não apresentaram diferenças de crescimento em relação às concentrações de $\mathrm{Fe}$ no meio GEL (Testemunha). Contudo, o crescimento foi maior quando se adicionou fosfato de $\mathrm{Fe}$, sugerindo $o$ aproveitamento do fósforo do fosfato. Aparente- mente, esta utilização não foi suficiente para formar uma área de solubilização, mas ela é indicativa de que existe algum processo de disponibilização.

Os quatro isolados restantes não solubilizaram em todos os níveis (Figura 3). O 154 e o 297 só solubilizaram fosfato de cálcio em concentrações de ferro bastante específicas. Isto parece não estar relacionado com o crescimento do microrganismo e sim às características do meio. $\mathrm{O}$ isolado 263 , também não teve seu crescimento afetado pelos teores de $\mathrm{Fe}$, só solubilizando em teores abaixo de $4,96 \mathrm{mg} \mathrm{L}^{-1} \mathrm{de}$ meio (Figura 3). Logo, a produção da substância solubilizadora estaria sendo realizada em condições de deficiência do elemento, sugerindo a possibilidade da produção de substâncias específicas no mecanismo de absorção de Fe (Masaoka et al., 1993; Wilhelm \& Trick, 1994; Shenker et al., 1995). Pode ser, ainda, que a deficiência de Fe estivesse afetando a atividade da aconitase, provocando acúmulo de ácidos (Marschner, 1995). O isolado 293 só solubilizou nos níveis mais altos de Fe. Em baixo nível $\left(0,45 \mathrm{mg} \mathrm{L}^{-1}\right)$, apresentou pouco crescimento e ausência de solubilização, demonstrando uma maior exigência de $\mathrm{Fe}$ em relação aos outros isolados (Figura 3).

Quanto ao Ca, a quantidade existente no meio com baixo teor $\left(230 \mathrm{mg} \mathrm{L}^{-1}\right)$ foi adequada para o crescimento dos isolados com colônias de diâmetro pequeno a médio (Figura 5). Para alguns destes isolados (293, 303, 263 e 264), o aumento do teor não afetou o tamanho da colônia, enquanto houve diminuição para outros $(238,05,154$ e 297), chegando a inibir o crescimento (85). Isto pode estar relacionado com a suscetibilidade à pressão osmótica do meio. De certa forma, a área solubilizada acompanhou o crescimento da maioria destes microrganismos, exceto os isolados 154 e 297, que solubilizaram em concentração bem específica $\left(2.800 \mathrm{mg} \mathrm{L}^{-1}\right)$, o isolado 303 , que solubilizou menos no nível baixo (230 $\left.\mathrm{mg} \mathrm{L}^{-1}\right)$, e o 264 , que não solubilizou no nível mais elevado (8.800 $\left.\mathrm{mg} \mathrm{L}^{-1}\right)$.

Para os isolados que normalmente apresentam amplo crescimento (310 B, 86, 142, 250 e $310 \mathrm{~A})$, as baixas concentrações de $\mathrm{Ca}\left(230\right.$ e $\left.900 \mathrm{mg} \mathrm{L}^{-1}\right)$ foram insuficientes para promover o crescimento 


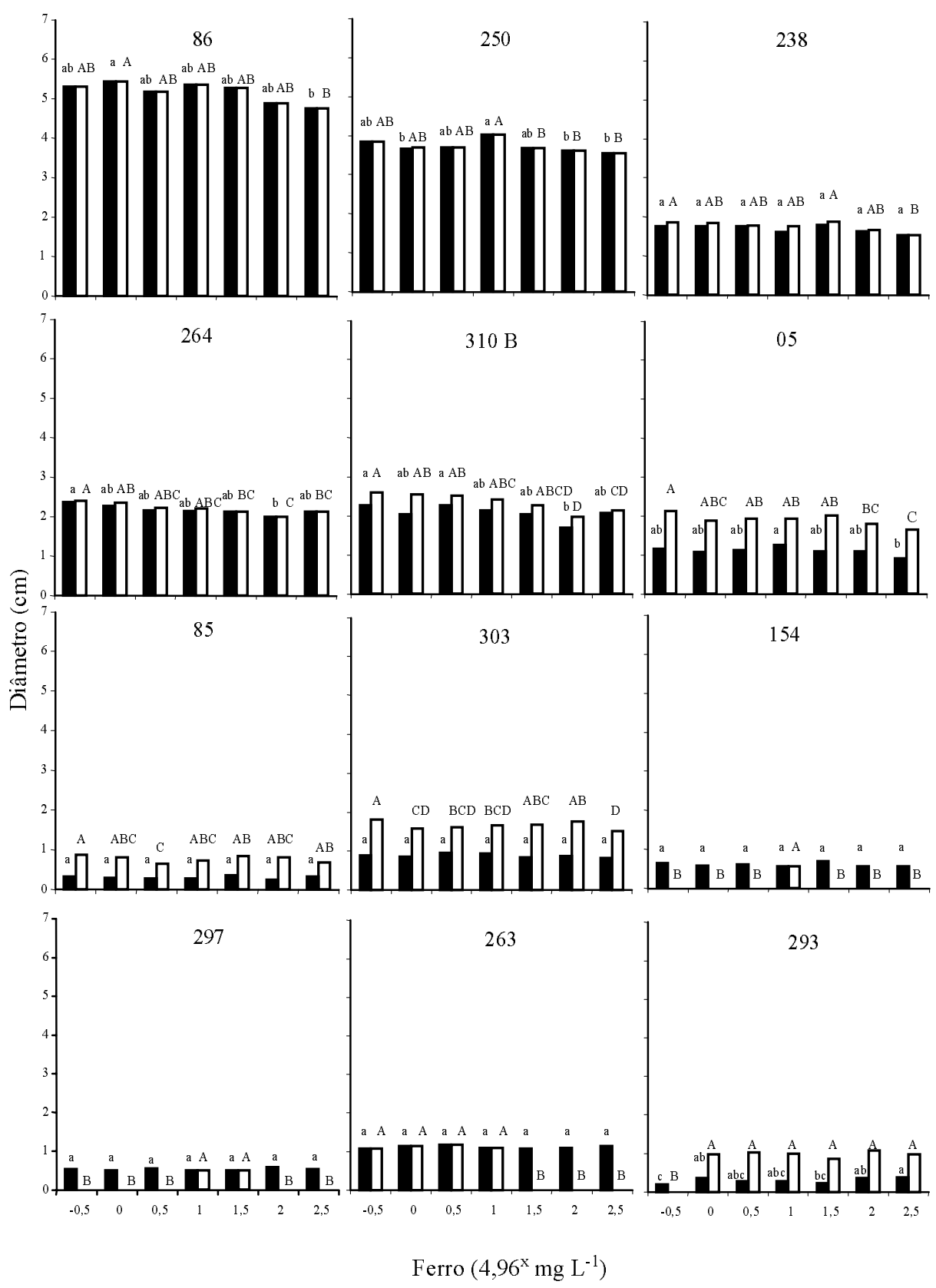

Figura 3. Efeito de teores de Fe no crescimento e na capacidade de solubilizar fosfato de cálcio dos isolados $86,250,238,264,310 \mathrm{~B}, 05,85,303,154,297,263$ e 293. Letras minúsculas comparam o diâmetro de colônia (ロ) e maiúsculas o do halo (口). 

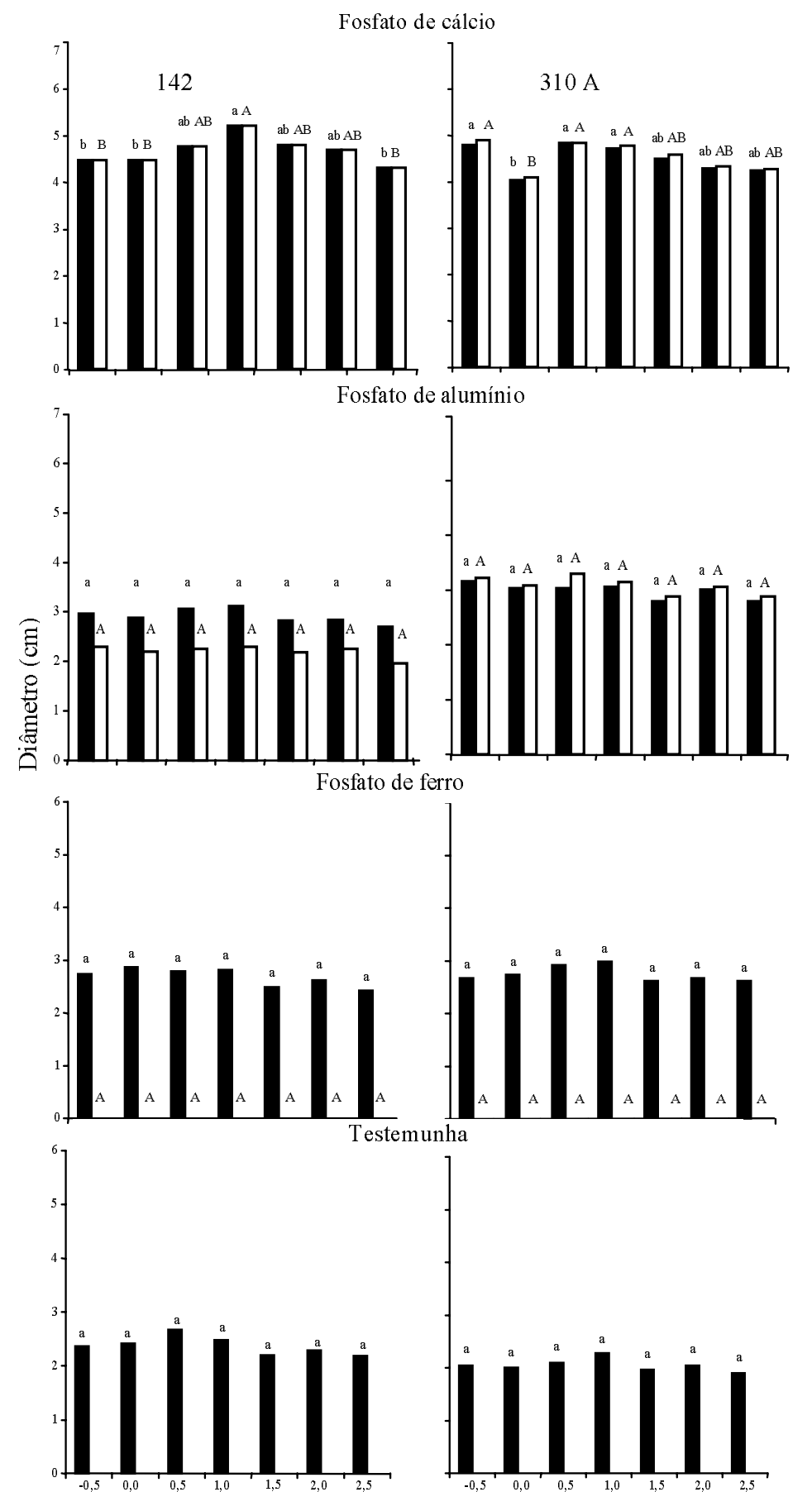

Ferro $\left(4,96^{\mathrm{x}} \mathrm{mg} \mathrm{L}^{-1}\right)$

Figura 4. Efeito de teores de Fe no crescimento e na capacidade de solubilizar fosfatos dos isolados 142 e $310 \mathrm{~A}$. Letras minúsculas comparam o diâmetro de colônia $(\boldsymbol{\square})$ e maiúsculas o do halo $(\square)$. 
(Figura 6). Isto aconteceu porque o Ca é essencial à atividade enzimática e à estrutura da membrana (Griffin, 1994), dificultando o crescimento com diminuição de sua disponibilidade. A partir do teor médio de $\mathrm{Ca}$ (2.800 $\left.\mathrm{mg} \mathrm{L}^{-1}\right)$, o isolado $310 \mathrm{~B}$ apresentou restrições de crescimento, possivelmente pela maior pressão osmótica. Nos níveis mais baixos, o tamanho da colônia e da área solubilizada foram similares. Com a restrição ao crescimento, apesar de a área solubilizada ter sido menor, proporcionalmente ela foi maior que a da colônia.

Os isolados 86 e 142 comportaram-se de maneira semelhante, apresentando decréscimo no tamanho da colônia a partir do 5 o nível de $\mathrm{Ca}\left(5.000 \mathrm{mg} \mathrm{L}^{-1}\right)$. O comportamento do halo e da colônia foram de certa forma os mesmos, sugerindo que a solubilização depende do crescimento (Figura 6).
Os isolados 310 A e 250 apresentam, no nível mais baixo, uma relação halo:colônia superior à dos demais níveis. Além do fosfato de $\mathrm{Ca}$, os isolados 142 e 310 A também solubilizaram fosfato de $\mathrm{Al}$, com resultados similares entre as duas fontes de fosfato.

$\mathrm{O}$ baixo suprimento de Ca parece estimular a produção de substâncias solubilizadoras, como podese deduzir pelo halo do isolado $310 \mathrm{~B}$ e pela relação halo:colônia nos isolados 250 e $310 \mathrm{~A}$. Cunningham \& Kuiack (1992) verificaram que Penicillium bilaji, em altas concentrações de $\mathrm{Ca}$, esporula e passa a alcalinizar o meio, ao passo que, em baixas concentrações, produz ácidos. Deve-se ainda considerar o Ca como nutriente, pois alguns organismos o requerem em maior quantidade (Marschner, 1995). Em baixos teores do nutriente, a retirada do $\mathrm{Ca}$ da solução pode resultar na dissolução do fosfato de $\mathrm{Ca}$ (Bekele et al., 1983; Robinson \& Syers, 1990).

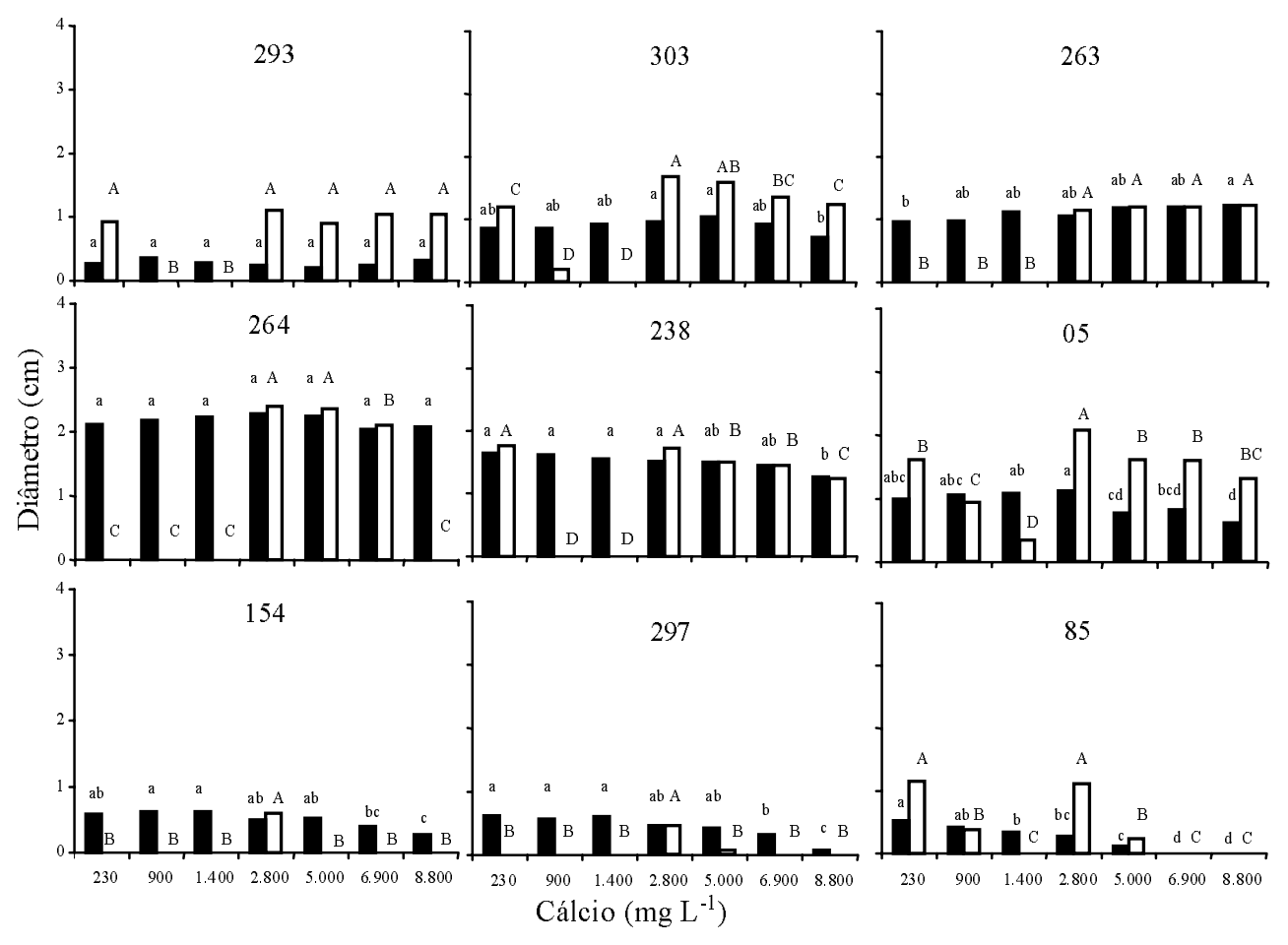

Figura 5. Efeito de teores de Ca no crescimento e na capacidade de solubilizar fosfato de cálcio dos isolados 293, 303, 263, 264, 238, 05, 154, 297 e 85. Letras minúsculas comparam o diâmetro de colô-

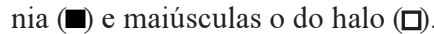




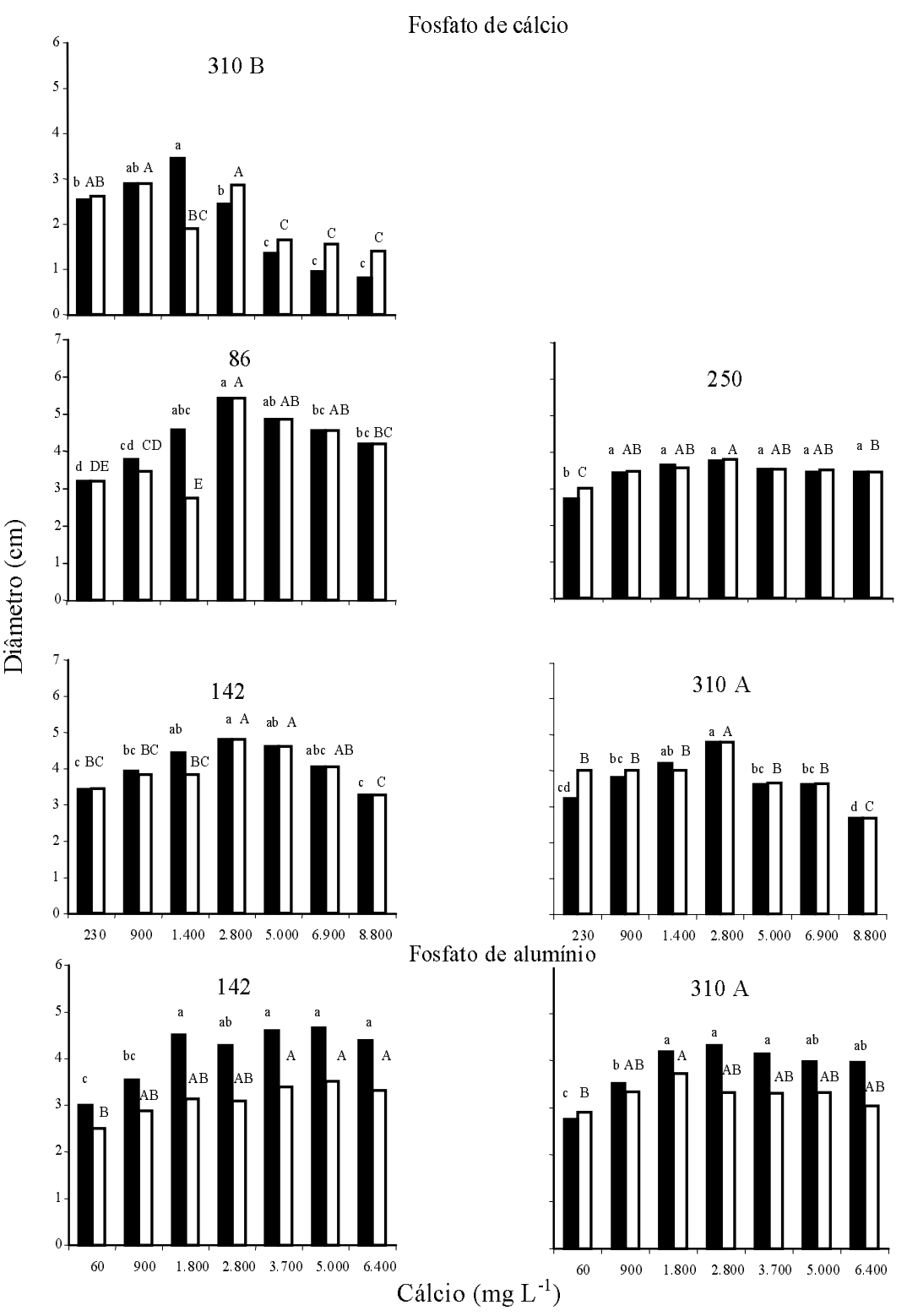

Figura 6. Efeito de teores de Ca no crescimento e na capacidade de solubilizar fosfato de cálcio e dos isolados 310 B, 86, 250, 142 e 310 A, e de alumínio, dos isolados 142 e 310 A. Letras minúsculas comparam o diâmetro de colônia (a) e maiúsculas o do halo ( $\square)$

O comportamento dos isolados em relação ao $\mathrm{K}$ foi semelhante ao obtido com $\mathrm{Ca}$. O aumento na concentração não provocou aumentos expressivos no crescimento dos microrganismos com colônias de tamanho pequeno a médio (Figura 7). Os isolados que produzem colônias grandes (Aspergillus) apresentaram efeitos de níveis de $\mathrm{K}$ sobre o crescimento (Figura 8). No nível mais baixo (130 $\left.\mathrm{mg} \mathrm{L}^{-1}\right)$, o isola- 
do 310 B apresentou o diâmetro da colônia igual ao do halo. Com a elevação dos teores de K para $700 \mathrm{e}$ $1.000 \mathrm{mg} \mathrm{L}^{-1}$, houve aumento do diâmetro da colônia proporcional ao halo, permanecendo a relação em torno de 1. A partir deste nível, o aumento da concentração provocou diminuição do diâmetro da colônia e do halo. Porém, esta diminuição não foi proporcional, sendo maior na colônia, o que resultou em uma relação superior a 1.

Os isolados 86 e 142 apresentaram uma relação constante. A área solubilizada e o diâmetro das colônias cresceram a partir do nível mais baixo até o nível médio de K, quando, então, tenderam a estabilizar. Os dados sugerem que o processo de solubilização é dependente das condições de crescimento e do produto elaborado pelo metabolismo normal do organismo, o que confirma as observações anteriores.

Os isolados 250 e 310 A apresentaram a mesma tendência de crescimento dos isolados anteriores. No entanto, o diâmetro do halo, na fonte com fosfato de Ca, foi superior ao da colônia, resultando em uma relação maior. Isto sugere uma restrição no crescimento, favorecendo a visualização do halo, ou uma maior produção ou liberação de metabólitos solubilizantes. Paris et al. (1996) verificaram que Pisolitus tinctorius e Paxillus involutus produziam mais oxalato em meio deficiente de potássio.
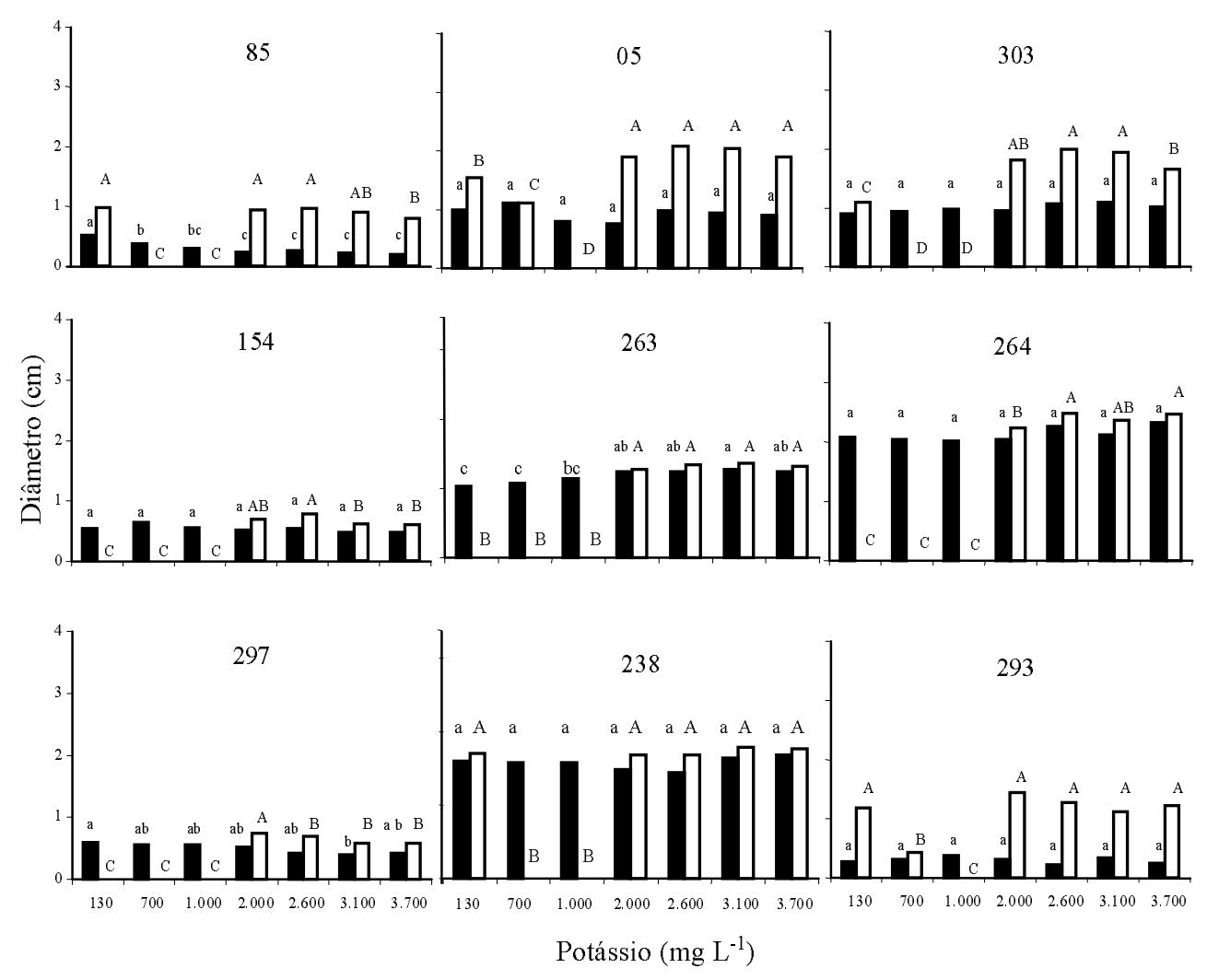

Figura 7. Efeito de teores de $\mathrm{K}$ no crescimento e na capacidade de solubilizar fosfato de cálcio dos isolados 85, 05, 303, 154, 263, 264, 297, 238 e 293. Letras minúsculas comparam o diâmetro de colônia $(\square)$ e maiúsculas o do halo $(\square)$. 
Fosfato de cálcio
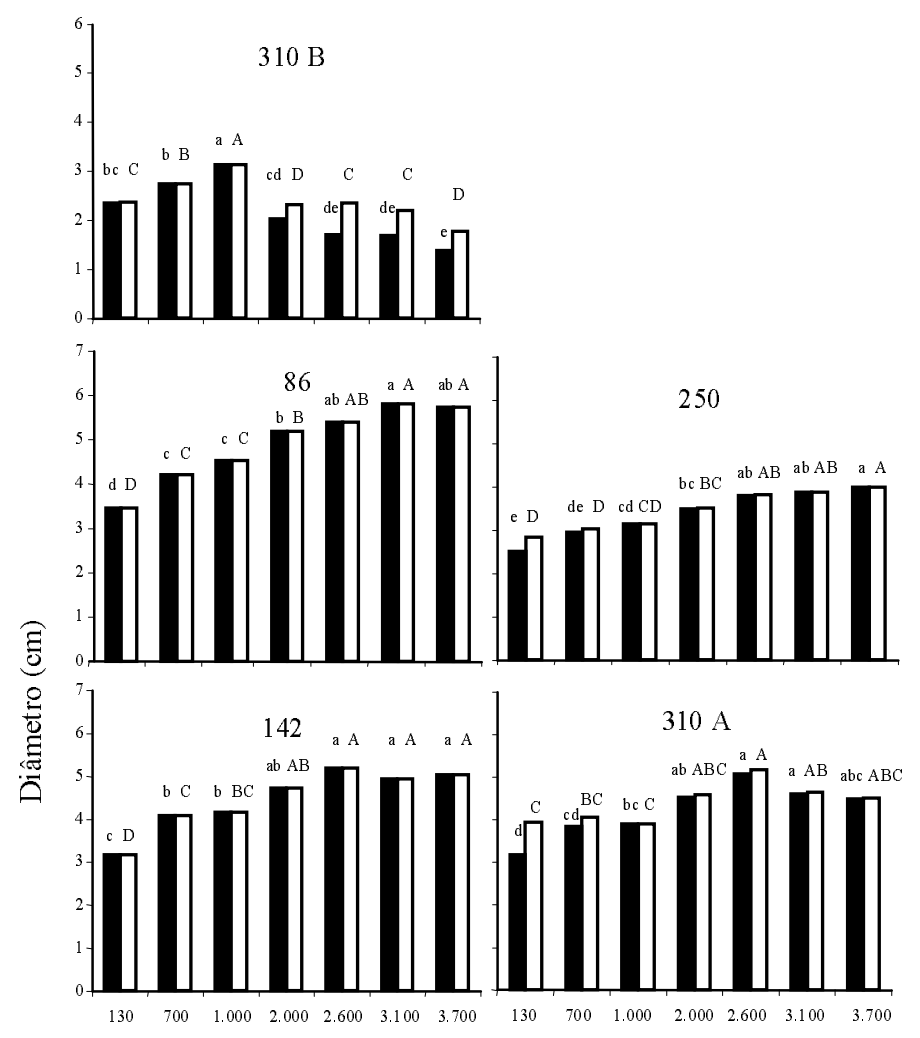

Fosfato de alumínio

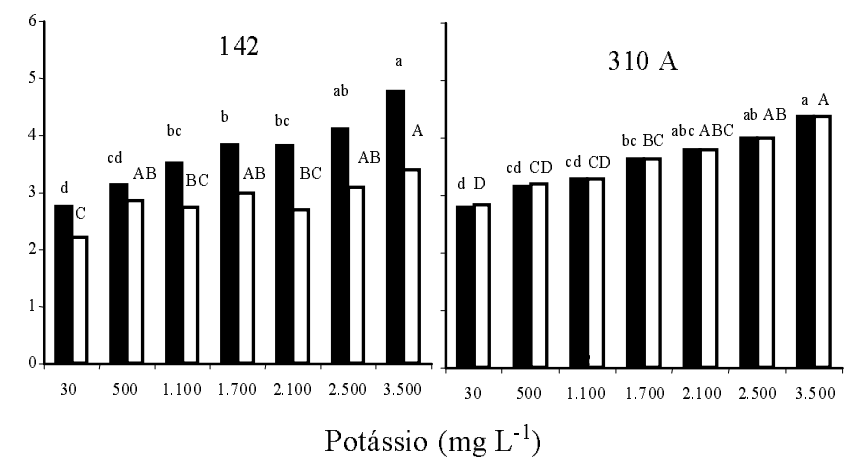

Figura 8. Efeito de teores de $\mathrm{K}$ no crescimento e na capacidade de solubilizar fosfato de cálcio e de alumínio dos isolados $310 \mathrm{~B}, 86,250$, 142 e 310 A. Letras minúsculas comparam o diâmetro de colônia (ם) e maiúsculas o do halo ( $\square$ ).

\section{Conclusões}

1. A capacidade e o potencial de solubilização dos microrganismos variam com os fatores nutricionais do meio.
2. A fonte de $\mathrm{N}$ afeta a capacidade e o potencial de solubilização, verificando-se um efeito positivo com fonte amoniacal e negativo com nítrica.

3. Baixos teores de $\mathrm{Ca}$ ou $\mathrm{K}$ reduzem o crescimento de isolados que apresentam grandes colônias 
(Aspergillus), e em alguns desses, aumentam o potencial de solubilização.

4. Variações nos teores de Fe não afetam a atividade solubilizadora da maioria dos microrganismos testados.

5. Diferenças na capacidade e no potencial de solubilização indicam alterações quantitativas ou qualitativas nos metabólitos produzidos, sugerindo a ocorrência de diferentes mecanismos de solubilização ou de processos com eficiência variável.

\section{Referências}

BAGG, A.; NEILANDS, J. B. Molecular mechanism of regulation of siderophore-mediated iron assimilation. Microbiological Reviews, Washington, v. 51, p. 509-518, 1987.

BEKELE, T.; CINO, B. J.; EHLERT, P.A. I.; MAAS, A.A van der; DIEST, A. van. An evaluation of plant-borne factors promoting the solubilization of alkaline rock phosphates. Plant and Soil, Dordrecht, v. 75, n. 3, p. 361378, 1983

CUNNINGHAM, J. E.; KUIACK, C. Production of citric and oxalic acids and solubilization of calcium phosphate by Penicillium bilaii. Applied and Environmental Microbiology, Washington, v. 58, n. 5, p. 1451-1458, 1992.

DARRAH, P. R. The rhizosphere and plant nutrition: a quantitative approach. Plant and Soil, Dordrecht, v. 155/ 156, p. 1-20, 1993.

FERNANDES, M. S.; SOUZA, S. R. A aquisição de N por plantas. In: SIMPÓSIO BRASILEIRO SOBRE NITROGÊNIO EM PLANTAS, 1., 1990, Itaguaí. Anais.. Itaguaí: Unicamp, 1990. p. 172-192.

GRIFFIN, D. H. Fungal physiology. 2. ed. New York: Wiley-Liss, 1994. 458 p.

HAYNES, R. J. Active ion uptake and maintenance of cation-anion balance: a critical examination of their role regulating rhizosphere $\mathrm{pH}$. Plant and Soil, Dordrecht, v. 126, n. 2 , p. 247-264, 1990.

ILLMER, P.; BARBATO, A.; SCHINNER, F. Solubilization of hardly-soluble $\mathrm{AlPO}_{4}$ with $\mathrm{P}$-solubilizing microorganisms. Soil Biology \& Biochemistry, Oxford, v. 27 , p. $265-270,1995$

ILLMER, P.; SCHINNER, F. Solubilization of inorganic phosphates by microorganisms isolated from forest soils
Soil Biology \& Biochemistry, Oxford, v. 24, n. 4, p. $389-$ 395, 1992

KIM, K. Y.; McDONALD, G. A.; JORDAN, D. Solubilization of hydroxyapatita by Enterobacter agglomerans and cloned Escherichia coli in culture medium. Biology and Fertility of Soils, Berlin, v. 24, n. 4 , p. $347-352,1997$

LAPEYRIE, F. Oxalate synthesis from soil bicarbonate by the mycorrhizal fungus Paxillus involutus. Plant and Soil, Dordrecht, v. 110, n. 1, p. 3-8, 1988.

LAPEYRIE, F.; RANGER, J.; VAIRELLES, D. Phosphate solubilizing activity of ectomycorrhizal fungi in vitro. Canadian Journal of Botany, Ottawa, v. 69, p. 342-346, 1991.

MacKAY,A. D.; SYERS, J. K.; TILLMAN, R. W.; GREGG, P. E. H. A simple model to describe the dissolution of phosphate rock in soils. Soil Science Society of America Journal, Madison, v. 50, n. 2, p. 291-296, 1986.

MARSCHNER, H. Mineral nutrition of higher plants. London: Academic Press, 1995. 889 p.

MASAOKA, Y.; KOJIMA, M.; SUGIHARA, S.; YOSHIHARA, T.; KOSHINO, M.; ICHIHARA, A. Dissolution of ferric phosphate by alfalfa (Medicago sativa L.) root exudates. Plant and Soil, Dordrecht, v. 155/ 156, p. 75-78, 1993

NEIDHARDT, F. C.; INGRAHQM, J. L.; SCHAECHTER, M. Physiology of the bacterial cell: a molecular approach. Sunderland: Sinauer Associates, 1990. 507 p.

PARIS, F.; BOTTON, B.; LAPEYRIE, F. In vitro weathering of phogopite by ectomycorrhizal fungi II: effect of $\mathrm{K}^{+}$and $\mathrm{Mg}^{++}$deficiency and $\mathrm{N}$ sources on accumulation of oxalate and $\mathrm{H}^{+}$. Plant and Soil, Dordrecht, v. 179, n. 1, p. 141-150, 1996.

ROBINSON, J. S.; SYERS, J. K. A critical evaluation of the factors influencing the dissolution of Gafsa phosphate rock. Journal of Soil Science, Oxford, v. 41, n. 4, p. 597 605,1990

SHENKER, M.; GHIRLANDO, R.; OLIVER, I.; HELMANN, M.; HADAR, Y.; CHEN, Y. Chemical structure and biological activity of a siderophe produced by Rhyzopus arrhizus. Soil Science Society of America Journal, Madison, v. 59, n. 3, p. 837-843, 1995.

Pesq. agropec. bras., Brasília, v. 36, n. 12, p. 1495-1508, dez. 2001 
SILVA FILHO, G. N. Solubilização de fosfatos pela microbiota do solo. Porto Alegre: UFRGS, 1998. 140 p. Tese de Doutorado.

SILVA FILHO, G. N.; VIDOR, C. Solubilização de fosfatos por microrganismos na presença de fontes de carbono Revista Brasileira de Ciência do Solo, Viçosa, MG, v. 24 , n. 2, p. 311-319, 2000.

SYLVESTER-BRADLEY, R.; ASAKAWA，N.; LA TORRACA, S.; MAGALHÃES, F. M. M.; OLIVEIRA, L. A.; PEREIRA, R. M. Levantamento quan- titativo de microrganismos solubilizadores de fosfatos na rizosfera de gramíneas e leguminosas forrageiras na Amazônia. Acta Amazônica, Manaus, v. 12, p. 15-22, 1982

TEDESCO, M. J.; GIANELLO, C.; BISSANI, C. A.; BOHNEN, H.; VOLKWEIS, S. J. Análise do solo, plantas e outros materiais. Porto Alegre: UFRGS, 1995. 174 p.

WILHELM, S. W.; TRICK, C. G. Iron-limited growth of cyanobacteria: multiple siderophe production is a common response. Limnology and Oceanography, Seattle, v. 39, n. 8, p. 1979-1984, 1994. 